\title{
Tratamento cirúrgico e conservador de cisto periapical de grande proporção: relato de caso
}

\author{
Maraísa Aparecida Pinto Resende* \\ Neuza Maria Souza Picorelli Assis* \\ Augusto César Sette-Dias** \\ Evandro Guimarães de Aguiar*** \\ Bruno Salles Sotto-Maior*
}

\begin{abstract}
RESUMO
O objetivo deste estudo foi descrever uma técnica de tratamento conservadora, cuja conduta preconizada foi a realização da descompressão seguida pela enucleação cística através do relato de um caso de cisto periapical de grande extensão. Paciente de 50 anos de idade, melanoderma, apresentando cisto periapical com aproximadamente $4,5 \mathrm{~cm}$ em seu maior diâmetro associado ao incisivo central inferior esquerdo. Na primeira etapa do tratamento, foi realizada descompressão cística e biopsia incisional, cujo exame histopatológico confirmou o diagnóstico clínico. Após 25 semanas de acompanhamento, houve uma considerável regressão do tamanho da lesão e a mesma foi enucleada sem comprometimento dos elementos dentários envolvidos e dos tecidos adjacentes. $\mathrm{O}$ relato de caso evidenciou que o tratamento cirúrgico e conservador pode ser um recurso em cistos periapicais de maior dimensão, sendo importante a cooperação do paciente no acompanhamento pós-operatório.
\end{abstract}

Palavras-chave: Cisto radicular. Cirurgia bucal. Descompressão cirúrgica.

\section{INTRODUÇÃO}

O cisto periapical é o cisto odontogênico mais comum, com uma prevalência que varia de $7 \%$ a $54 \%$ de todas as imagens radiolúcidas periapicais (NEVILLE et al., 2009; VASCONCELOS et al., 2012). Este cisto é do tipo inflamatório e a descamação de seu revestimento epitelial originado dos retos epiteliais de Malassez mantém o processo inflamatório local ativo. Geralmente, os cistos periapicais são assintomáticos e têm crescimento lento, mas algumas vezes podem atingir grandes proporções (VASCONCELOS et al., 2012; ARAÚjO et al., 2013; BAVA et al., 2015).

Geralmente, quando possuem dimensões menores são descobertos através de uma radiografia de rotina, porém quando atingem maiores dimensões podem ser identificados pela presença de tumefação, sensibilidade, assimetria, mobilidade e deslocamento dos dentes (VASCONCELOS et al., 2012; ARAÚJO et al., 2013; KADAM et al., 2014; BAVA et al., 2015; HUG et al., 2016). Radiograficamente verifica-se uma imagem radiolúcida unilocular bem definida circundando o ápice de um dente (BERAR et al., 2016).

Podem ser indicados para o tratamento dos cistos periapicais tratamentos conservadores através do tratamento endodôntico do dente desvitalizado com ou sem apicetomia e tratamentos cirúrgicos como a extração do elemento dentário seguida de curetagem periapical, marsupialização ou ainda a enucleação cística (VASCONCELOS et al., 2012; HUANG et al., 2016; SALARIA; KAMBA; GHUMAN, 2016). Sendo que a cirurgia periapical é mais indicada para lesões que excedem $2 \mathrm{~cm}$ de diâmetro e para aquelas associadas a dentes nos quais não é viável o tratamento endodôntico convencional (NARULA et al., 2011).

O exame histopatológico é indicado para descartar outras possíveis doenças como, por exemplo: ceratocisto odontogênico, lesão central de células gigantes, ameloblastoma e outros cistos e tumores odontogênicos (NEVILLE et al., 2009); além de confirmar o diagnóstico clínico (BERAR et al., 2016).

Diante do exposto, o presente trabalho tem por objetivo apresentar um caso clínico com um tratamento inicialmente conservador e finalizado por meio de enucleação cirúrgica, evidenciando a importância da biópsia incisional para guiar o profissional na conduta terapêutica mais adequada para o paciente, evitando assim mutilações desnecessárias.

*Universidade Federal de Juiz de Fora, Faculdade de Odontologia, Departamento de Clínica Odontológica - Juiz de Fora, MG. maraisa_rc@hotmail.com **Centro Universitário Newton Paiva, Departamento de Odontologia - Belo Horizonte - MG.

***Universidade Federal de Minas Gerais, Faculdade de Odontologia, Departamento de Cirurgia Oral - Belo Horizonte, MG. 


\section{Relato de Caso Clínico}

Paciente de 50 anos, melanoderma, sexo feminino, sem alterações sistêmicas, apresentou-se na Clínica de Cirurgia da Faculdade de Odontologia da Universidade Federal de Minas Gerais com a queixa principal de que seu dente estava movimentando-se há aproximadamente 12 meses. Ao exame clínico apresentou tumefação em região lingual e anterior da mandíbula e deslocamento vestibular acentuado do incisivo central inferior direito (Figura 1).

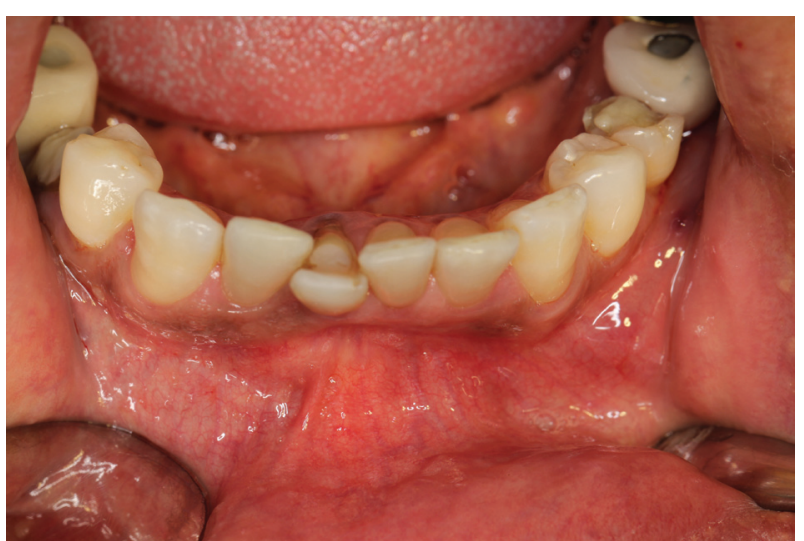

Figura 1: Deslocamento vestibular do incisivo central inferior direito e leve tumefação lingual.

Fonte: Os autores (2015).

Durante a avaliação radiográfica, constatou-se a presença de imagem radiolúcida unilocular extensa de 4,5 x $2 \mathrm{~cm}$ de extensão, bem delimitada por halo radiopaco, estendendo-se da região de segundo prémolar inferior direito à região do canino inferior esquerdo, circundando os ápices dos mesmos, com perda de lâmina dura, deslocamento dos dentes adjacentes e reabsorção radicular externa. (Figura 2).
Os elementos dentários responderam ao teste de sensibilidade pulpar ao frio, exceto o incisivo central inferior, que apresentava seu conduto tratado endodonticamente. Foi realizada punção aspirativa por agulha (1,20 x $2518 \mathrm{G} 1)$, tendo como resultado a presença de líquido amarelado com traços de sangue, com características de líquido cístico (Figura 3).

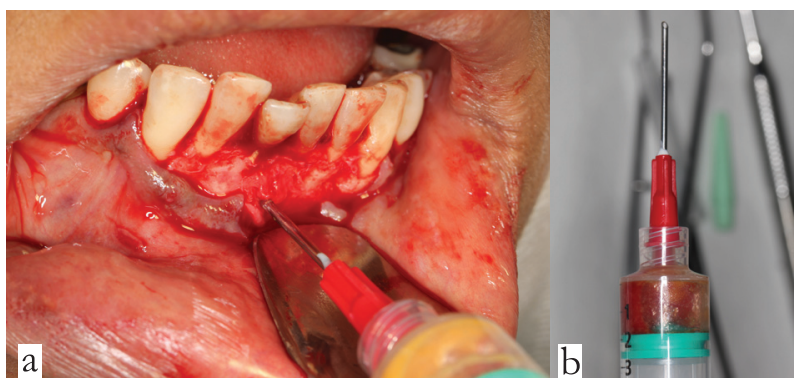

Figura 3: (a) Punção aspirativa por agulha grossa. (b) Presença de líquido amarelo citrino.

Fonte: Os autores (2015).

Em seguida foi realizada uma biópsia incisional através da osteotomia da parede vestibular e remoção de um fragmento de $0,75 \times 0,5 \mathrm{~cm}$ da parede cística para posterior avaliação histopatológica (Figura 4).

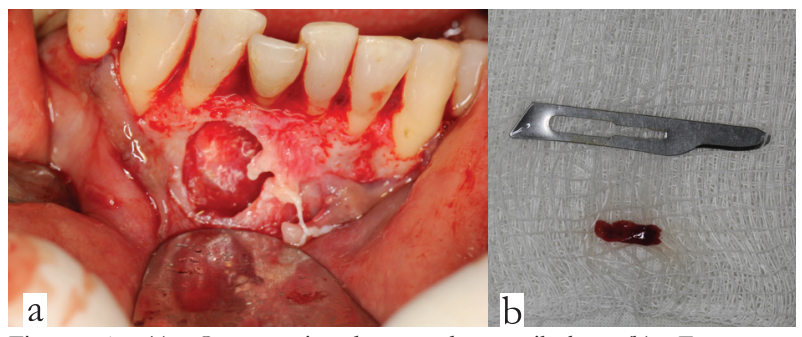

Figura 4: (a) Osteotomia da parede vestibular. (b) Fragmento anatomopatológico removido do tecido de revestimento da parede cística. Fonte: Os autores (2015).

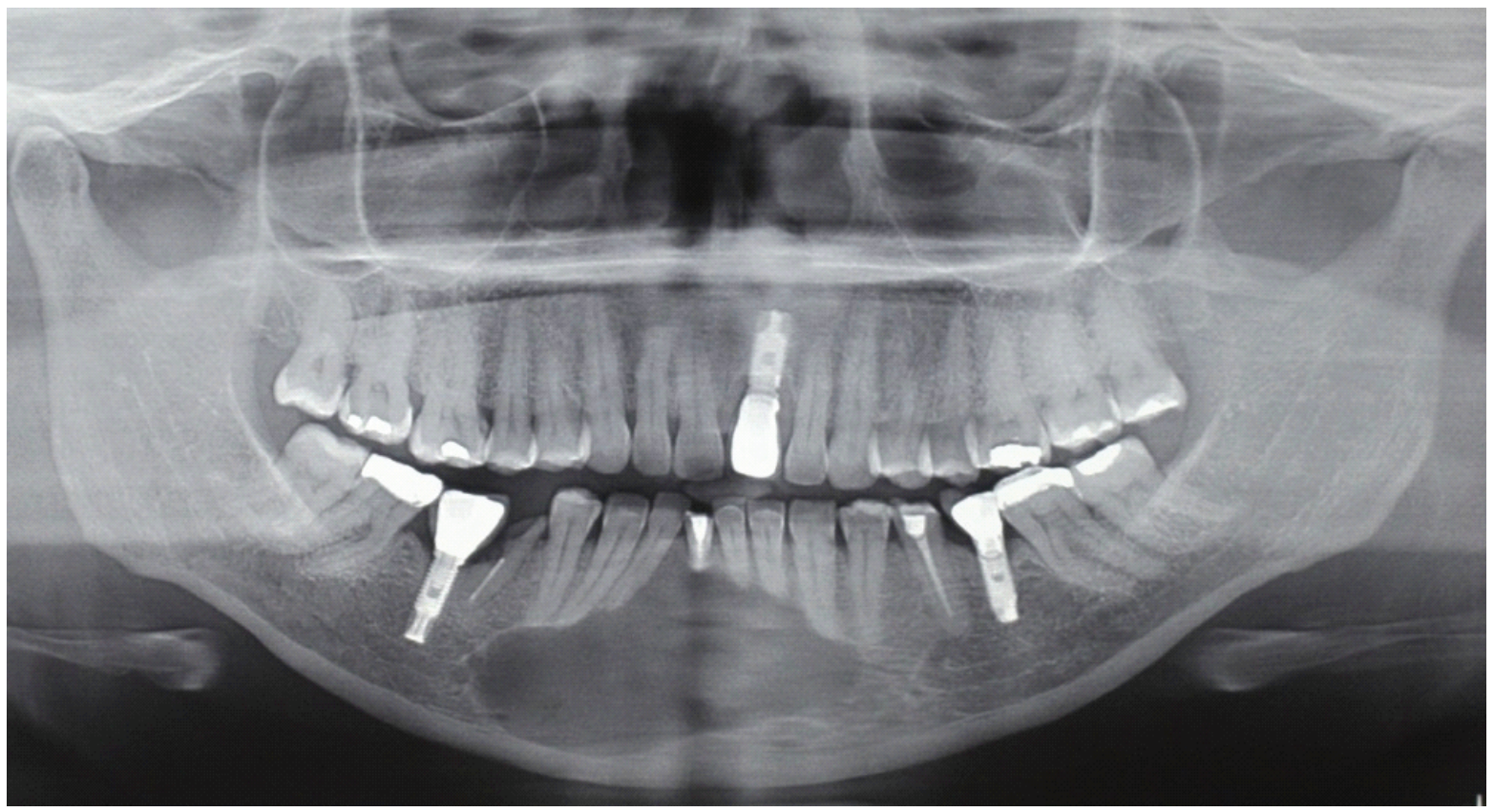

Figura 2: Radiografia panorâmica inicial.

Fonte: Os autores (2015). 
A cavidade cirúrgica foi irrigada em abundância, e suturas simples interpapilares foram realizadas com fio de Nylon 5-0. Uma cânula de polipropileno foi adaptada no interior da cavidade cística até a cavidade oral. A cânula foi fixada aos dentes adjacentes à abertura através de fio de aço (Aciflex 0). A paciente foi orientada a realizar irrigações com soro fisiológico duas vezes ao dia através da mesma por quatro meses e depois manter o controle pós-operatório por mais tempo caso fosse necessário. (Figura 5).

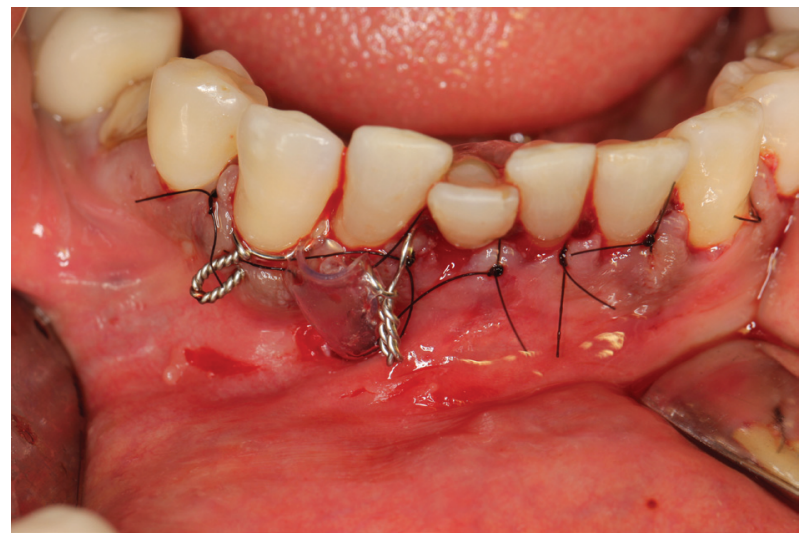

Figura 5: Suturas e cânula de polipropileno em posição. Fonte: Os autores (2015).

O resultado do exame histopatológico foi de cavidade cística revestida por epitélio estratificado pavimentoso não ceratinizado; áreas focais de espongiose; cápsula constituída por tecido conjuntivo fibroso; focos de escasso infiltrado inflamatório mononuclear; e áreas de hemorragia, compatível com o diagnóstico de cisto inflamatório.

No controle pós-operatório de 25 meses, a radiografia panorâmica evidenciou reparo ósseo, cânula de polipropileno em posição, cisto radicular de pequena proporção envolvendo a raiz do incisivo central inferior direito (Figura 6).

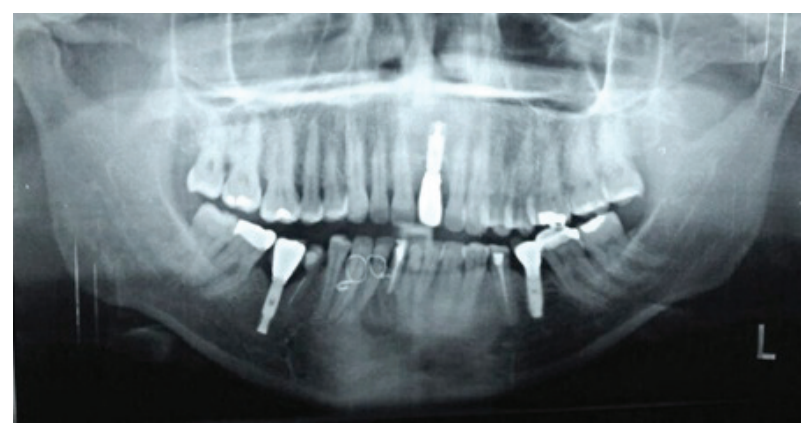

Figura 6: Radiografia panorâmica pós-operatória de 25 meses. Fonte: Os autores (2015).

Nesta etapa, a enucleação e curetagem do cisto periapical foram realizadas e o ato cirúrgico aconteceu sem intercorrências, não houve lesão ao nervo mentual e aos dentes adjacentes.

Em controle pós-operatório do segundo ato cirúrgico de 12 meses, a paciente apresentou-se sem queixas. Ao exame clínico observou-se incisivo alinhado ao arco dentário, ausência de mobilidade, ausência de tumefações e presença de cicatriz na região mucogengival onde se encontrava o dreno (Figura 7).

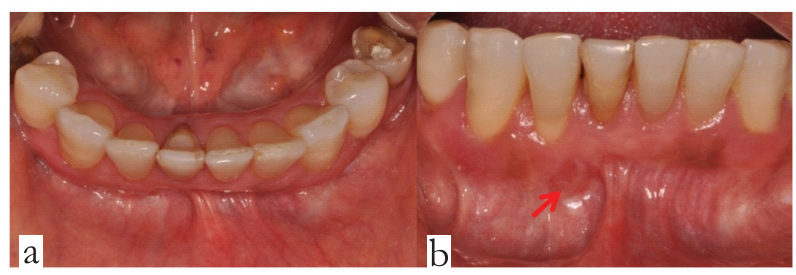

Figura 7: (a) Vista oclusão mostrando alimento dental e ausência de tumefação. (b) Vista vestibular mostrando presença do incisivo central e cicatriz na linha mucogengival (seta).

Fonte: Os autores (2018).

Ao exame radiográfico (Figura 8), observou-se ausência do dreno e ausência de lesão associada ao incisivo central inferior direito, que se encontra em posição.

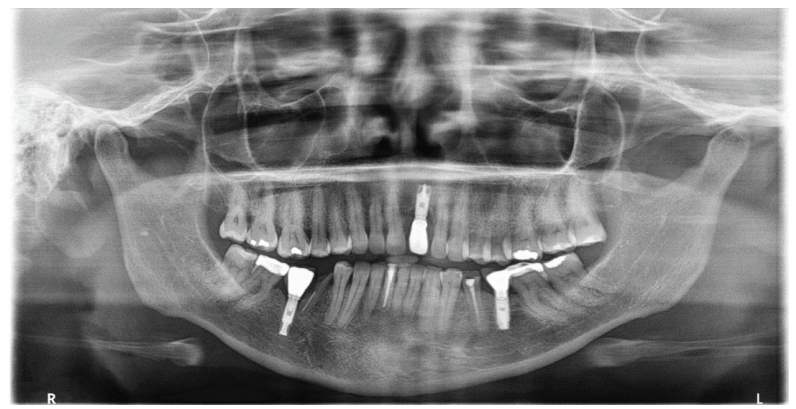

Figura 8: Radiografia panorâmica de 12 meses após a segunda intervenção cirúrgica.

Fonte: Os autores (2018).

\section{DisCUSSÃo}

O cisto periapical é uma entidade patológica de origem inflamatória mais comum dos cistos odontogênicos representando de 40 a $85 \%$ de todas as lesões apicais (OCHSENIUS et al., 2007, TORTORICI et al., 2008, NANAMI et al., 2008, SHARIFIAN et al., 2011; VASCONCELOS et al., 2012). A grande disparidade quanto à prevalência está relacionada, muito provavelmente, ao rigor dos critérios de diagnóstico utilizados em um estudo específico. Quando critérios rigorosos são utilizados, a prevalência dos cistos periapicais parece ser de apenas quase 15\% (NEVILLE et al., 2009).

A maioria dos cistos periapicais cresce lentamente e não atinge grandes dimensões. De acordo com Nobuhara e del Rio (1993), um diagnóstico clínico preliminar de cisto periapical pode ser feito se tiverem as seguintes características clínicas: a lesão periapical envolver um ou mais dentes com necrose pulpar; a lesão for maior do que $2 \mathrm{~cm}$ de diâmetro ou área transversal de $200 \mathrm{~mm}^{2}$; apresentar cor palha no líquido de aspiração ou drenagem; e o fluido conter cristais de colesterol. No presente relato foram observadas 
tais características que auxiliou no diagnóstico clínico da lesão.

Foi observado radiograficamente uma extensa área radiolúcida, medindo $4,5 \mathrm{~cm}$ em seu maior diâmetro e estendendo-se dos elementos dentários 45 até o 33. Também se verificou clinicamente mau posicionamento dentário dos elementos 11 ao 13, tumefação e ausência de sintomatologia. Esses achados corroboram em grande parte com os casos de cistos periapicais de grandes extensões relatados na literatura (VASCONCELOS et al., 2012; ARAÚJO et al., 2013; KADAM et al., 2014; BAVA et al., 2015; HUG et al., 2016; TORRES-LAGARES et al., 2011).

As opções terapêuticas para as lesões císticas periapicais variam desde o tratamento não cirúrgico, correspondendo ao tratamento endodôntico convencional até o tratamento cirúrgico, como curetagem e enucleação cística. Existe também a possibilidade da variação dessas técnicas, como a descompressão e marsupialização prévia à enucleação, assim como a combinação das mesmas (cirurgia parendodôntica) (BAVA et al., 2015; PEKER et al., 2016). Segundo SAKKAS et al. (2007) e BRANDT FILHO et al. (2010) os tratamentos cirúrgicos que podem ser realizados como alternativas em lesões radiculares extensas incluem a curetagem, enucleação, descompressão, marsupialização e ressecção em bloco.

A descompressão da cavidade cística é indicada em casos de lesões císticas de grandes proporções (TAKASE et al., 1996; SAKKAS et al., 2007; BRANDT FILHO et al., 2010). Esta técnica diminui ou elimina a possibilidade de comprometer a vitalidade de dentes adjacentes ou causar danos a nervos (BAVA et al., 2015; HUANG et al., 2016; PEKER et al., 2016; SALARIA; KAMRA; GHUMAN, 2016; TORRESLAGARES et al., 2011). Porém pode apresentar desvantagens, tais como a necessidade de cooperação do paciente por um longo período, inflamação da mucosa alveolar, persistência do defeito cirúrgico no local de inserção do dreno, possibilidade de desenvolvimento de infecção aguda ou crônica na lesão durante o tratamento e submergência do tubo no tecido (PENUMATSA et al., 2013; TAKASE et al., 1996).

No referido relato de caso, foi realizada a técnica com abordagem conservadora. Foi realizada a descompressão com a inserção de um dreno no interior da cavidade cística para permitir sua redução. Após 25 meses de acompanhamento e colaboração do paciente, que manteve irrigação com soro fisiológico diariamente através da cânula de descompressão, a lesão regrediu de $4,5 \mathrm{~cm}$ para $1 \mathrm{~cm}$, passando a envolver apenas a raiz do incisivo central inferior direito.

Uma segunda intervenção cirúrgica com a enucleação da lesão foi realizada com êxito. Isso se deve aos benefícios que a descompressão proporcionou, distanciando das proximidades do nervo mentual e das raízes dos dentes adjacentes, favorecendo, assim, a remoção completa da lesão e reduzindo o grau de recidiva e danos a estruturas anatômicas vitais.

Segundo NETO et al. (2004), os cistos de origem inflamatória não recidivam após um tratamento adequado e ocasionalmente podem ocorrer cicatrizes fibrosas em vez de neoformação óssea. Em relação ao caso clínico, o paciente se encontra em acompanhamento de 12 meses pós-operatório e não houve sinais de recidiva e o reparo ósseo pôde ser verificado, assim como nos trabalhos de POZZER et al., (2009); TORRES-LAGARES et al., (2010) e VASCONCELOS et al. (2012).

O sucesso no tratamento de cistos periapicais de grande extensão pode ser alcançado através de um correto e minucioso diagnóstico, planejamento de tratamento adequado, acompanhamento clínico e radiográfico e cooperação do paciente (BAVA et al., 2015; HUANG et al., 2016; PEKER et al., 2016; SALARIA; KAMRA; GHUMAN, 2016; TORRESLAGARES et al., 2011).

\section{Conclusão}

A técnica da descompressão cística com a utilização de um dreno na região buco-maxilo-maxial mostrou-se satisfatória e eficaz como método auxiliar conservador de tratamento. Este procedimento promoveu uma significativa redução da lesão, possibilitando a posterior enucleação da lesão sem provocar danos às estruturas anatômicas próximas e dentes envolvidos, além de ter evitado uma cirurgia extensa. 


\title{
Surgical and conservative treatment of large periapical cyst: case report
}

\begin{abstract}
The aim of this study was to describe a conservative treatment technique, the recommended therapeutic of which was performed decompression and followed by cystic enucleation through the report of a large periapical cyst case. A 50-year-old melanoderma patient presented a periapical cyst with approximately $4,5 \mathrm{~cm}$ in its largest diameter associated with the left lower central incisor. In the first stage of the treatment, cystic decompression and incisional biopsy were performed; whose histopathological examination confirmed the clinical diagnosis. After follow-up, there was a considerable regression of the size of the lesion and it could be enucleated without compromising the dental elements involved and adjacent tissues. The case report showed that surgical and conservative treatment may be a resource in larger periapical cysts, and patient cooperation in postoperative follow-up is very important.
\end{abstract}

Keywords: Radicular cyst. Oral Surgery. Descompression Surgical.

\section{REFERÊNCIAS}

BAVA, F.A.; UMAR, D.; BAHSEER, B.; BAROUDI, K. Bilateral radicular cyst in mandible: an unusual case report. Journal of International Oral Health, v. 7, n. 2, p. 61-63, feb. 2015.

BERAR, A.M.; BONDOR, C.I.; MASTROS, L. CÂMPIAN, R.S. Radiological, histological and immunohistochemical evaluation of periapical inflammatory lesions. Romanian Journal of Morphology and Embryology, v. 57, n. 2, p. 419-425, 2016.

BRANDT-FILHO, S.H.O.; MARZOLA, C.; TOLEDO-FILHO, J.L.; PEREIRA, L.C.; PASTORI, C.M.; ZORZETTO, D.L.G.; CAPELARI, M.M.; TOLEDO, G.L. Técnica da descompressão para o tratamento cirúrgico dos cistos da cavidade bucal. Revista ATO, v.5, n. 1, p.254-266, 2010.

LIN, L.M.; HUANG, G.T-J. Patobiologia do Periápice. In: HARGREAVES, K.M.; COHEN, S. Caminhos da Polpa. 10.ed. Rio de Janeiro: Elsevier Editora Ltda, 2011. cap. 14, p. 502-505.

HUANG, H.Y.; CHEN, Y.K.; KO, E.C.; CHUANG, F.H.; CHEN, P.H.; CHEN, C.Y.; WANG, W.C. Restrospective analysis of nonendodontic periapical lesions in a population of Taiwanese patients. Clinical Oral Investigations, v. 21, n. 6, p. 2077-2082, jul. 2016.

HUG, J.K.; YANG, D.K.; JEON, K.J.; SHIN, S.J. Progression of periapical cystic lesion after incomplete endodontic treatment. Restorative Dentistry \& Endodontics, v. 41, n. 2, p. 137-142, may. 2016.

KADAM, N.S.; ATAIDE, I.N.; RAGHAVA, P.; FERNANDES, M.; HEDE, R. Management of large radicular cyst by conservative surgical approach: a case report. Journal of Clinical and Diagnostic Research, v. 8, n. 2, p. 239-241, Feb. 2014.

NANAMI，R.; SAMPAIO, C.; OLIVETE, J.; PIZZATTO, E.; MORESCA, R.; GIOVANINI, A.F. Prevalência de cistos maxilares diagnosticados em centro de referencia brasileiro.
Revista Sul-Brasileira de Odontologia, v. 6, n. 2, p. 143-146, mai. 2008.

NARULA, H.; AHUJA, B.; YELURI, R.; BALIGA, S.; MUNCHI, A.K. Conservative no-surgical management of an infected radicular cyst. Contemporary Clinical Dentistry, v. 2, n. 4, p. 368-371, oct./dec. 2011.

NETO, M.N.; DANESI, C.C.; UNFER, D.T. Contribuição ao estudo do cisto radicular revisão da literatura. Revista Saúde, v. 30, n. 1-2, p. 90-99, 2004.

NEVILLE, B.W.; DAMM, D.D.; ALLEN, C.M., BOUQUOT, J.E. Doenças da Polpa e do Periápice. In: Patologia oral \&Maxilofacial. 3.ed. Rio de Janeiro: Guanabara Koogan, 2009. cap.3, p. $129-134$.

NOBUHARA, W.; DEL REIO, C. Incidence of periradicular panthoses in endodontic treatment failures. Journal of Endodontics, v. 18, n. 6, p. 315-318, jun. 1993.

OCHSENIUS, G.; ESCOBAR, E.; GODOY, L.; PEÑAFIEL, C. Odontogenic cysts: analysis of 2.944 cases in Chile. Medicina Oral, Patología Oral y Cirugía Bucal, v. 12, n. 1, p. 85-91, mar. 2007.

PEKER, E.; OGUTLU, F.; KARACA, I.R.; GULTEKIN, E.S.; CAKIR, M. 5 year retrospective study of biopsied jaw lesions with the assessment of concordance between clinical and histopathological diagnoses. Journal of Oral and Maxillofacial Pathology, v. 20, n.1, p. 78-85, jan./apr. 2016.

PENUMATSA, N.V.; NALLANCHAKRAVA, S.; MUPPA, R.; DANDEMPALLY, A.;PANTHULA, P. Conservative approach in the management of radicular cyst in a child: case report. Case Reports in Dentistry, v. 17, p.1-3, feb. 2013.

POZZER, L.; JAIMES, M.; NETTO, H.D.M.C.; OLATE, S.; BARBOSA, J.R.A. Cistos odontogênicos em crianças: análise da 
descompressão cirúrgica em dois casos. Revista de Cirurgia e Traumatologia Buco-Maxilo-Facial, v.9, n. 2, p. 17-22, abr./ jun. 2009.

SAKKAS, N.; SCHOEN, R.; SCHULZE, D.; OTTEN, J.E.; SCHMELZEISEN, R. Obturator after marsupialization of a recurrence of a radicular cyst of the mandible. Oral Surgery, Oral Medicine, Oral Pathology, Oral Radiology, and Endodontics, v. 103, n.1, p. 16-18, jan. 2007.

SALARIA, S.K.; KAMRA, S.; GHUMAN, S.K. Nonsurgical endodontic therapy along with minimal invasive treatment utilizing Bhasker's hypothesis for the management of infected radicular cystic lesion: A rare case report. Contemporary Clinical Dentistry, v. 7, n. 4, p. 562-565, oct./dec. 2016.

SERRANO-GIMÉNEZ, M.; SÁNCHEZ-TORRES, A.; GAYESCODA, C. Prognostic fators on periapical surgery: a systematic review. Medicina Oral, Patología Oral y Cirugía Bucal, v. 20, n. 6, p. 715-722, nov. 2015.

SHARIFIAN, M.J.; KHALILI, M. Odontogenic cysts: a retrospective study of 1227 cases in an Iranian population from 1987 to 2007. Journal of Oral Science, v. 53, n. 3, p. 361-367, sep. 2011.
TAKASE, T.; WADA, M.; NAHAGAMA, F.; YAMAZAKI, M. Treatment of large radicular cysts by modified marsupialization. The Journal of Nihon University School of Dentistry, v. 38, n. 3.4, p. 161-168, dec. 1996.

TORRES-LAGARES, D.; SEGURA-EGEA, J.J.; RODRÍGUESCABALLERO, A.; LLAMAS-CARRERAS, J.M.; GUTIÉRREZPÉREZ, J.L. Treatment of a large maxillary cyst with marsupialization, descompression, surgical endodontic therapy and enucleation. Journal of Canadian Dental Association, v. 77 , n. 87 , jun. 2011.

TORTORICI, S.; AMODIO, E.; MASSENTI, M.F.; BUZZANCA, M.L.; BURRUANO, F; VITALE, F. Prevalence and distribution of odontogenic cysts in Sicily: 1986-2005. Journal of Oral Science, v. 50, n. 1, p.15-18, mar. 2008.

VASCONCELOS, R.G.; QUEIROZ, L.M.G.; ALVES JÚNIOR, L.C.; GERMANO, A.R.; VASCONCELOS, M.G. Abordagem Terapêutica em Cisto Radicular de Grandes Proporções - Relato de Caso. Revista Brasileira de Ciências da Saúde, v. 16, n. 3, p. 467-474, 2012. 